\title{
Development of a Patient Safety Checklist for Use during Fluoroscopic or Image-guided, Minimally-invasive Procedure in the Department of Radiology, Philippine General Hospital
}

\author{
Ryan Jason DL. Urgel, MD and Imarzen V. Elepano, MD \\ Department of Radiology, College of Medicine and Philippine General Hospital, University of the Philippines Manila
}

\begin{abstract}
Objective. This study aimed to develop a patient-safety checklist for use during fluoroscopic- or image-guided minimally-invasive procedures in the Department of Radiology, Philippine General Hospital.

Methodology. A comparison of the Radiological Patient Safety System (RADPASS) and the Cardiovascular and Interventional Society of Europe (CIRSE) checklists was done. Together with the knowledge of the workflow and through observation of the different procedures in the department, a checklist was developed to suit the appropriate hospital setting. This developed checklist was tested in several procedures, and was evaluated and modified during trial testing.
\end{abstract}

Result. A patient safety checklist for minimally-invasive and fluoroscopic procedures in the Department of Radiology, Philippine General Hospital was developed through the analysis of the workflow of the department, and observation during the data gathering and trial testing phases.

Conclusion. This simple tool was developed to ensure that all the necessary details before a procedure have been addressed. It has been made as simple as possible, to make it user-friendly. The developed checklist is a step forward in promoting and ensuring the safety of patients undergoing fluoroscopic and minimally-invasive procedures in the Department of Radiology, PGH.

Key Words: patient safety, checklist, radiology

\section{INTRODUCTION}

Patient safety should always be upheld in everything a doctor does - may it be from a simple palpation and auscultation, to an invasive open heart surgery, or to even something in between, such as diagnostic or minimallyinvasive procedures. The safety and well-being of patients should always come first - the benefit of the procedure must outweigh the risks - and respecting the patient's decision to either push-through with or forego the procedure. Patient safety is related to the occurrence of adverse events, defined

Poster presented in the $2^{\text {nd }}$ Patient Safety Congress on March 28-29, 2019, at the Philippine International Convention Center, Manila Philippines.

Corresponding author: Ryan Jason DL. Urgel, MD, FPCR

Department of Radiology

Philippine General Hospital

University of the Philippines Manila

Taft Avenue, Manila 1000, Philippines

Email: urgelmd@gmail.com as untoward medical occurrence that may result in death or persistence of significant disability or incapacity. ${ }^{1}$ Although this term is mainly used for drug reactions, it has been loosely adapted for any problems that may arise during diagnostic imaging and procedures. In a systematic review in 2007, adverse events during hospital admission were found in one out of 10 patients. A substantial proportion of these adverse events is preventable and is often surgery- or drug-related. ${ }^{2}$ 
The World Health Organization (WHO) has long developed a Safe Surgery Checklist to address safety concerns during surgical procedures. This checklist has been used by surgeons and operating suite staff to minimize any errors that may arise from as simple as premedication errors, to as grave as wrong laterality, organ, or patient. This checklist identified three phases of an operation, and with each phase, a coordinator must confirm to the team that all the tasks were completed before continuing with the procedure. ${ }^{3}$

Similar checklists have been developed by international Interventional Radiology societies for use in their procedures, usually those done in the Catheterization Laboratory. Examples of these are the Radiological Patient Safety System $(\text { RADPASS })^{4}$ and Cardiovascular Interventional Society of Europe (CIRSE) IR checklists. ${ }^{5}$ The RADPASS was developed based on observing the daily practice in a tertiary referral center in the Netherlands. After its validation, it was used successfully in reducing deviations from the optimal process in their radiology departments. The CIRSE IR Checklist was developed based on the WHO Surgical Safety Checklist and RADPASS.

There are similarities between fluoroscopic- or imageguided, minimally-invasive procedures and other surgical and interventional procedures. Generally, the similarities point to patients being placed in a procedure with risks that may affect their well-being.

In computed tomography- or ultrasound-guided procedures such as biopsies, local anesthesia is given by the doctor after patient preparation to minimize local site pain. Thereafter, a needle is used to obtain specimens for laboratory or histopathological studies. In fluoroscopic procedures, a continuous flow of radiation is given to the patient to acquire the images that radiologists need for a sound radiologic diagnosis. Dynamic fluoroscopic studies use contrast media for visualization of internal organs to be evaluated. All the above procedures carry risks to the patient, no matter how minor these are.

At the time of writing, no checklist has been developed for such procedures in the Department of Radiology, Philippine General Hospital (PGH). It is important to develop such a checklist to ensure the safety of patients who will undergo minimally-invasive or fluoroscopic procedures in the said department. The purpose of this checklist is to reduce the deviations from established departmental guidelines, and to help decrease the occurrence of preventable errors that can lead to patient harm.

\section{MATERIALS AND METHODS}

This was an observational, descriptive study done in the Department of Radiology, PGH. The process was divided into three parts:

Data Gathering Phase: Data for the planned patient safety checklist was based on the review of the RADPASS and the CIRSE IR checklists, identifying similarities between these and determining the important points in the checklists that were usable in the current set-up in the Department of Radiology, PGH. Information from the knowledge of the workflow in the department and of the different imageguided and fluoroscopic procedures was also taken into consideration in the development of the checklist. A week of observation of the different fluoroscopic and imageguided procedures was also done. During the observation of procedures, the interaction of the primary investigator with the patients and radiology trainees was ensured to be minimal. It was made certain that the performers of the procedures, mainly the radiology trainees, were not aware of being observed. This was accomplished through some random visits to the different procedure rooms (fluoroscopy, CT scan or ultrasound), making casual conversations with the trainees and not possessing any material that can imply doing an observation. This set-up for the observation of procedures may have helped lessen the inherent biases noted in observational studies.

Checklist Development Phase: Based on the data gathered, a checklist was developed to suit to the appropriate hospital setting and workflow of the medical and paramedical staff in the Department of Radiology, PGH.

Trial Phase: The developed checklist was tested in several procedures in the department. It was accomplished by the projected end-users - the radiology trainees. These trainees are involved in almost every step of the procedure, ensuring the performance of pre-procedural checks and administering post-procedural care among patients who underwent certain procedures in the department. It was tested in elective procedures - at least 5 in fluoroscopy, 5 in $\mathrm{CT}$ scan, and 5 in ultrasound. This is an arbitrary number to check if the developed checklist is still in need of modifications, and not to assess the validity of the checklist. Modification of the checklist was done throughout the course of the trial phase, as needed.

\section{Ethical Consideration}

The protocol for this study was submitted to the UP Manila Research Ethics Board (UPMREB) PGH Review Panel for ethics review, waiving of informed consent, and approval. Although patient's details were needed for the developed patient safety checklist during the trial phase, patient identifiers were intentionally left blank for patient confidentiality. No additional risk was incurred by patients and medical and paramedical staff during the data gathering and trial phases of the study. No external source of funding was made available. There are no conflicts of interests.

\section{Limitations of the Study}

As this study was based on the workflow of the Department of Radiology, PGH, and the dynamics of its staff and patients, the developed patient safety checklist may not be applicable to similar procedures done by other clinical services or by other hospitals. The number of procedures 
where the checklist was tested was small, which cannot be the used as basis for testing its validity.

\section{RESULTS AND DISCUSSION}

The need for a safety checklist prior to performing procedures in the Catheterization Laboratory has already been realized by some Interventional Radiology societies, as an increasing number of morbidities and mortalities were seen among patients who had a procedure in the hospital. Minimally-invasive procedures can pose as a threat to patients, if certain details have been neglected, be it intentional or otherwise. Simple fluoroscopic procedures can also lead to similar harm. A checklist can help lessen or even eliminate such harm, as this can serve as a reminder to the doctors on what to check prior to the procedure. Such an activity may decrease the occurrence of guideline deviations, with proper documentation.

The Department of Radiology, PGH has a guideline being followed by its doctors that is aligned to ensuring patient safety and proper workflow within the department. However, such steps during an actual procedure were not properly documented. Admittedly, some steps were overlooked unintentionally (but are later realized). This is where the importance of a checklist comes in.

Through observation of the actual workflow in the Department of Radiology and knowledge of the different minimally-invasive and fluoroscopic procedures being done in the department, certain information were either retained or modified in the previously mentioned checklists (the RADPASS and the CIRSE IR checklists). Tabulation and analysis of the details within the checklists were done.

A patient safety checklist was developed based on the data gathered. Details included in the checklist were divided into five parts: (1) General Patient Information, (2) Preprocedural check, (3) Time-out, (4) After the procedure, and (5) Check and Balance.

General Patient Information: It is important to include certain patient identifiers such as the patient's name, age, sex, and case number. This was to ensure that the procedure to be performed is to the right patient. The type and date of the procedure was likewise deemed important, especially for the census of the department. A question on whether it is the patient's first time to undergo such procedure, or a follow-up or repeat procedure was placed to ensure that if a similar procedure has been done, comparison can be made, especially for fluoroscopic studies.

Pre-procedural check: This refers to information that may be relevant to the radiologist performing the procedure. This information may lead to the deferral of the procedure, if after screening the patient, the radiologist deemed the risk of the procedure to be far greater than the benefits. In this portion of the checklist, a question regarding having the right diagnostic request form is included to comply with the guidelines of the department. Questions regarding any history of contrast allergy or the need for contrast-induced nephropathy (CIN) prophylaxis have been included for fluoroscopic procedures requiring the need for contrast. If there was a history of allergy to contrast media and no prophylactic measures have been done, the radiologist may defer the contrast-requiring fluoroscopic procedure. Any patient with deranged creatinine or estimated glomerular filtration rate (GFR) who is to undergo a similar procedure, with no medical clearance or did not have prophylaxis for possible CIN, may have his or her procedure deferred. Overall, the questions that were deemed important to be included in the checklist were those that can lead to deferring the procedure if not ultimately safe for the patient.

Time-out: It is important to identify that the right procedure is to be done to the right patient, in the right site/ organ. Ensuring that all records brought by the patient were essential, we determine if there are any recent events that can be detrimental to the patient if the procedure pushes through. These include recent laboratory test results and any available imaging done to the patient. A question regarding ensuring patient's compliance to the pre-procedural orders was included. Similar to the WHO Surgical Safety Checklist, introducing the team who will do the procedure was vital to alleviate any fears or concerns from the patient, which usually arises from not knowing who will do the procedure.

After the procedure: It is important that post-procedural notes or orders should be given and written in the patient's chart. In a busy setting, this step can sometimes be overlooked by the radiologist. As such, the question on post-procedural notes was deemed important to be included, as a reminder to them. Any tissue sample or specimen, as in the case of biopsies done under computed tomography or ultrasound guidance, should be properly labeled and sent to the laboratory, or given to the patient and watcher. Documentation of such activity is important to reduce any errors that may arise from either mislabeled or misplaced specimens. A question on whether an imaging is needed after the procedure was likewise placed to ensure that such procedure will be ordered in the patient's chart, to monitor any post-procedural complications not evident in the immediate post-procedure period. For fluoroscopic procedures, a question on the total radiation time was asked, to document the radiation exposure of the patient. This information may lead to decreasing any future radiation exposures, in order to comply with the maximum allowable radiation exposure of the patient. It must be remembered that excessive radiation to a patient can lead to complications later on in his or her life.

Check and Balance: The developed checklist followed the style in the RADPASS. After the "Pre-procedural check" part, a question on whether to continue or postpone the procedure was asked. If the latter is selected, the radiologist must write down the reason for deferring the procedure. The radiologist is then asked to affix his or her signature for proper documentation. At the end of the checklist, the radiologist 
Case Number:

Patient Name:

Age/Sex:
Date of Procedure:

Procedure Done:

Prior procedures done/date:

\section{PATIENT SAFETY CHECKLIST FOR ULTRASOUND/CT-GUIDED AND FLUOROSCOPIC PROCEDURES}

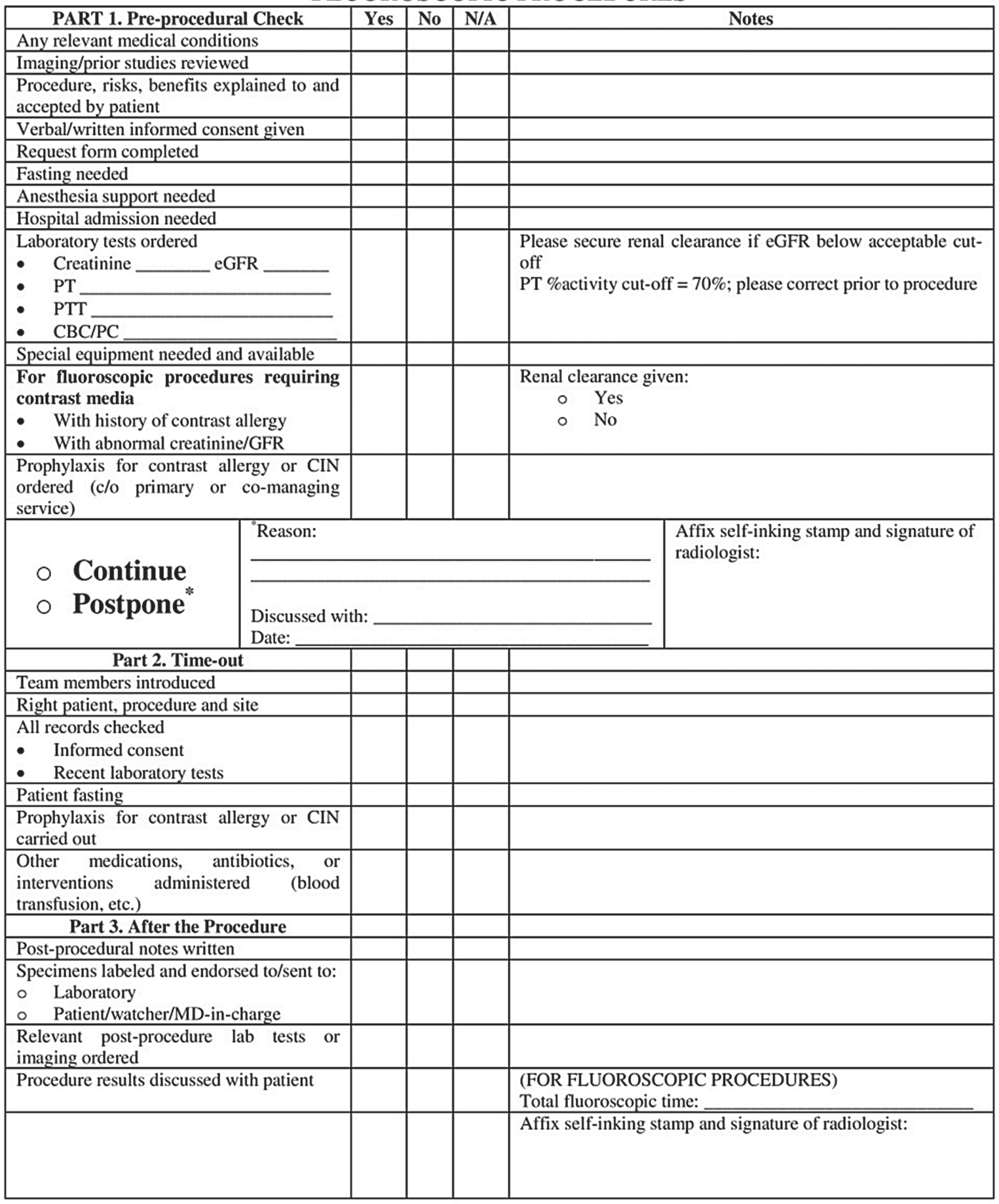

Figure 1. Developed patient safety checklist for the Department of Radiology, PGH. (Scaled to fit for publication. Actual size will fit a whole A4 sheet of paper, ensuring that all details are clear during its use.). 
is likewise asked to affix his or her signature to ensure that all questions have been answered properly.

To provide an initial assessment of the checklist regarding its context, content, structure, and usability in the Department of Radiology, PGH, the end-users of the checklist, the radiology trainees, were asked to answer the "Checklist for formatting checklists" adapted from the Western Michigan University. ${ }^{6}$ This was done during the trial phase of the study.

Based on the answers of the end-users, a title was deemed necessary. As such, a title was placed in the topmost part of the checklist, to avoid any possible confusion as to what the checklist is and its purpose. Although not written at the start of the checklist, an instruction was given to the endusers regarding how to use the checklist, and whom to ask if there is any confusion on how to use it. This was done to limit the checklist to a single page, as more than that can lead to the thought of a lengthy and tiresome paperwork.

The radiology trainees who answered the checklist felt that it is complete and technically correct. The terms used were consistent, and deemed precise, as the items in the checklist pointed to what is really needed. Checkpoints were seen in place, with notes as to what the user of the checklist has to do. Although the acronyms used, such as CBC, PT/ PTT, and the like, were not spelled out, no confusion arose from this because these acronyms were universally accepted ones. Only one action was addressed per item in the checklist, and as such, did not lead to uncertainties on how to use it.

The structure of the checklist likewise did not lead to any problems on its use. The checklist was designed in such a way that actions were grouped according to the time by which the user needs to perform the action relative to the procedure. It was intentional to not place a number before the action to be done, as the user can perform the task in any order he or she wishes to. The items in the checklist were grouped and boxed accordingly; different font sizes and typefaces were used to imply whether the action is an item under one of the parts of the checklist, or whether the item is a checkpoint.

The final developed checklist, after the trial use and minor revisions, is presented in Figure 1.

\section{CONCLUSION AND RECOMMENDATIONS}

The checklist developed for use in minimally-invasive and fluoroscopic procedures in the Department of Radiology, PGH was made as simple as possible, to ensure that future users can use it without hesitations and uncertainties. Relevant and important questions were included in the checklist and were easily answerable by ticking the user's response. Although the checklist is well-formatted with good content and context based on the feedback of the endusers, the validity of the developed checklist was not assessed in this study, given the small number of procedures where it was trial tested. A validation study may be done in the future to ensure that it will not cause any delays in and will improve the delivery of patient care. The developed checklist is a step forward in promoting and ensuring the safety of patients undergoing fluoroscopic and minimally-invasive procedures in the Department of Radiology, PGH.

\section{Acknowledgment}

The author wishes to acknowledge the help of the residents of the Department of Radiology, PGH during the implementation of this study. The information they provided were helpful in the completion of this study.

\section{Statement of Authorship}

Both authors participated in data collection and analysis, and approved the final version submitted.

\section{Author Disclosure}

Both authors declare no conflicts of interest.

\section{Funding Source}

No external funding acquired for this study.

\section{REFERENCES}

1. World Health Organization. Definition of Terms: Serious adverse event or reaction [Internet]. WHO website [cited 2015 April 1]. Available from: http://www.who.int/medicines/areas/quality_safety/ safety_efficacy/trainingcourses/definitions.pdf

2. De Vries EN, Ramrattan MA, Smorenburg SM, Gouma DJ, et al. The incidence and nature of in-hospital adverse events: a systematic review. Qual Saf Health Care [Internet]. 2008 [cited 2015 April 1];17:216-223. Available from http://www.ncbi.nlm.nih.gov/pmc/ articles/PMC2569153/

3. World Health Organization. WHO surgical safety checklist and implementation manual [Internet]. WHO website [cited 2015 April 1]. Available from http://www.who.int/patientsafety/safesurgery/ ss_checklist/en/

4. Koetser IC, de Vries EN, van Delden OM, Smorenburg SM, et al. A checklist to improve patient safety in interventional radiology. Cardiovasc Intervent Radiol [Internet]. 2013 [cited 2015 April 1]36:312-19.

5. Lee MJ, Fanelli F, Haage P, Hausegger K, et al. Patient safety in interventional radiology: A CIRSE IR checklist. Cardiocasv Intervent Radiol [Internet]. 2012 [cited 2015 April 1]35:244-46. Available from https://www.ncbi.nlm.nih.gov/pmc/articles/PMC3298647/

6. Bichelmeyer BA. Checklist for formatting checklists. Evaluation Checklists Project [Internet]. Western Michigan University [cited 2015 July 1]. Available from https://www.wmich.edu/sites/default/ files/attachments/u350/2014/formattingchecklist.pdf 